\title{
Crecimiento Poblacional y Productividad de la Microalga Nativa Chlorella peruviana bajo Diferentes Salinidades
}

\author{
Population Growth and Productivity of the Native Microalga Chlorella \\ peruviana under DifFerent SALINITIES
}

\author{
Mauro Mariano Astocondor ${ }^{1,4}$, Egma Mayta Huatuco ${ }^{2}$, Haydee Montoya Terreros ${ }^{3}$, \\ Ronald Tarazona Delgado ${ }^{1,3}$
}

\section{Resumen}

El objetivo del estudio fue evaluar cinco niveles de salinidad sobre el crecimiento y productividad de Chlorella peruviana Chacon, cepa nativa de la laguna salina Mellisera, Chilca, Perú. Se utilizaron cinco tratamientos de acuerdo al nivel de salinidad: T1, 38\%o; $\mathrm{T} 2$, 44\% ; T3, 59\%; T4, 88\% ; y T5, 176\% , con una temperatura de $27 \pm 2{ }^{\circ} \mathrm{C}$ y una intensidad de luz de $1250 \pm 10 \mathrm{~lx}$. Cada tratamiento se realizó por triplicado en matraces de 250 , 500 y $1000 \mathrm{ml}$. Los cultivos se realizaron con medio f/2 (Guillard), aireación constante y fotoperiodo de 24:00 $\mathrm{h}$. Los resultados indicaron que T1 presentó mejores resultados en densidad $\left(42 \pm 2.6 \times 10^{6} \mathrm{cel} / \mathrm{ml}\right)$, biomasa seca $(397.9 \pm 2.3 \mathrm{mg} / \mathrm{l})$ y clorofila $(80.2 \pm 2.3 \mathrm{mg} / \mathrm{l})$, pero con rendimientos adecuados en los niveles superiores de salinidad. Se concluye que el crecimiento y productividad de $C$. peruviana presenta una tendencia decreciente con relación al nivel de salinidad.

Palabras clave: ecología; fisiología; producción; cultivo; algas clorofíceas

\section{ABSTRACT}

The aim of the study was to evaluate five salinity levels on the growth and productivity of Chlorella peruviana Chacon, a native strain of the Mellisera salt lagoon in Chilca, Peru. Five treatments were used according to the salinity level: T1, 38\%o; T2, 44\%; T3, $59 \%$; T4, 88\%o; and T5, 176\%o, with a temperature of $27 \pm 2{ }^{\circ} \mathrm{C}$ and a light intensity of

\footnotetext{
${ }^{1}$ Laboratorio de Investigación Dulceacuícola y de Cordados, ${ }^{2}$ Laboratorio de Virología Clínica y Molecular, Facultad de Ciencias Biológicas, Universidad Nacional Mayor de San Marcos, Lima, Perú

${ }^{3}$ Laboratorio de Simbiosis Vegetal, Museo de Historia Natural, Universidad Nacional Mayor de San Marcos, Lima, Perú

${ }^{4}$ E-mail:mmarianoa@unmsm.edu.pe,mgmarianoa@gmail.com
}

Recibido: 15 de febrero de 2017

Aceptado para publicación: 27 de julio de 2017 
$1250 \pm 10 \mathrm{~lx}$. Each treatment was done in triplicate in flasks of 250, 500 and $1000 \mathrm{ml}$. Cultures were performed with f/2 medium (Guillard), constant aeration and photoperiod of 24:00 h. The results indicated that $\mathrm{T} 1$ showed better results in density $(42 \pm 2.65 \times 106 \mathrm{cel} / \mathrm{ml})$, dry biomass $(397.9 \pm 2.3 \mathrm{mg} / \mathrm{l})$ and chlorophyll $(80.2 \pm 2.3 \mathrm{mg} / \mathrm{l})$, but with adequate yields at higher levels of salinity. It is concluded that the growth and productivity of C. peruviana presents a decreasing trend in relation to the level of salinity.

Key words: ecology; physiology; production; cultivation; chlorophyceae algae

\section{INTRODUCCIÓN}

El género Chlorella Beijerinck, 1890, comprende el grupo de microalgas Cloroficeas, que se caracterizan por ser cosmopolitas y frecuentes en lagos alcalinos, marinos y lagunas costeras (Hernández y Labbe, 2014). Chlorella es una de las microalgas con abundante contenido de proteína y que desde los tiempos de la II Guerra Mundial (Álvarez y Gallardo, 1989) fue utilizado como suplemento alimenticio por su fácil reproducción y potencial nutritivo, capaz de estimular, aunque de forma parcial, el buen desarrollo de los procesos vitales del organismo humano. Se cultiva de forma intensiva y el sistema por lote es el más utilizado a gran escala por su bajo riesgo de contaminación y fácil implementación (Infante et al., 2012). La creciente popularidad de Chlorella entre los consumidores y las obvias necesidades de producción de alimentos, ponen de manifiesto la importancia de buscar alternativas de producción de este grupo en ambientes marinos y continentales (Emad et al., 2015)

Muchas especies de Chlorella son eurihalinas, pero los límites de tolerancia de las especies varían considerablemente, sobre todo las que se encuentran distribuidas en ecosistemas costeros como las lagunas salinas y pozas efemerales, las cuales están sujetas a cambios de salinidades, donde adquiere importancia su capacidad de adaptación para sobrevivir en tales ambientes (Kirst, 1989; Adenan et al., 2013; Emad et al., 2015).
La producción de biomasa en Chlorella vulgaris, Chlorella sorokiniana y Chlorella sp en aguas marinas y continentales no está bien definida y se requiere precisar los límites de salinidad que soportan estas especies, según lo indican algunos investigadores (Spolaore et al., 2006; Mathad y Hiremath, 2009; Krienitz et al., 2015).

Se ha demostrado que el factor salinidad disminuye los procesos metabólicos y la replicación celular y que, además, existe una correlación inversa entre la salinidad y la densidad celular. Por otro lado, el conocimiento de la dinámica de crecimiento de microalgas (tasa de inoculación, densidad celular en el tiempo, máxima tasa de crecimiento, tiempo de generación y producción de biomasa), productividad (producción de biomasa seca y clorofilas) y su manipulación, es indispensable para generar un sistema viable de producción, tanto técnico como económico (Graeff, 2003; Rodrigues y Belli-Filho, 2004; Yamamoto et al., 2004; Rodas et al., 2012).

En la actualidad se considera a $C$. peruviana una especie nativa poco estudiada, con un alto potencial para ser usado como fuente de alimento vivo para larvas de crustáceos, moluscos y peces e inclusión en dietas comerciales extruidas de inicio para peces, así como en biotecnología algal, como compuestos antivirales para el virus dengue. Los antecedentes que existen de la cepa en mención, aislada de las lagunas salinas costera, se limitan a su hábitat y descripciones taxonómicas (Vincens et al., 1971; Chacón, 
1980; Montoya y Espinoza, 1985; CalvoPérez y Molinari, 2015).

El objetivo del presente estudio fue evaluar cinco concentraciones de salinidades sobre el crecimiento y productividad de $C$. peruviana, con el fin de conocer el nivel de salinidad óptimo que permita el mayor crecimiento, producción de biomasa y clorofila en menor tiempo en condiciones de laboratorio con un sistema de cultivo discontinuo.

\section{Materiales y Métodos}

Los cultivos se realizaron en el Laboratorio de Investigaciones Dulceacuícolas y de Cordados de la Facultad de Ciencias Biológicas de la Universidad Nacional Mayor de San Marcos, Lima, Perú.

\section{Recolecta y Aislamiento de la Cepa}

Se utilizó una cepa de microalga Chlorella peruviana G. Chacón proveniente del cepario del laboratorio en mención y cultivado en medio f/2 (Guillard, 1975). La cepa original fue obtenida de muestras del fitoplancton de la laguna salina la «Mellisera» (12 $54^{\prime} 20^{\prime \prime}$ LS y $76^{\circ} 72^{\prime} 52^{\prime \prime}$ LW) en el distrito de Chilca, Lima, y aislada a partir de un cultivo masivo mediante técnica de rayado en placa y el método de la pipeta capilar para obtener un cultivo axénico.

\section{Cultivo de Microalgas}

Para determinar la salinidad óptima en el desarrollo de C. peruviana en cuanto a crecimiento (densidad celular) y productividad (biomasa y clorofila) se planteó evaluar cinco tratamientos de acuerdo al nivel de salinidad: 38\% (T1), 44\% (T2), 59\%о (T3), $88 \%$ (T4), y $176 \%$ (T5), con una temperatura de $27 \pm 2{ }^{\circ} \mathrm{C}$ y una intensidad de luz de $1250 \pm 101 x$. Asimismo, se consideraron tres repeticiones por tratamiento. Los valores de las salinidades se basaron en las recomen- daciones de Kessler y Huss (1992) y Huss et al. (1999). Las concentraciones de sales requeridas se obtuvieron mediante mezcla de agua destilada y sal comercial común (Alveal, 1995), corroboradas con un refractómetro calibrado (ATAGO S/Mill). La salinidad inicial fue de $38 \%$ debido a que es el promedio de salinidad del lugar de estudio.

Se utilizó el sistema de cultivo discontinuo (no hay adición de nutrientes posterior a la inoculación) con medio f/2 (Guillard, 1975) preparado con agua de mar esterilizado y microfiltrado proveniente del Laboratorio de Cultivos Marinos del Instituto del Mar del Perú.

Para evaluar el efecto de los niveles de salinidad sobre las diversas fases de crecimiento de $C$. peruviana, se realizaron cultivos en tubos de ensayo con tapa esterilizada y aireación en medio de cultivo $\mathrm{f} / 2$ para una primera aclimatación a la variación de los niveles de salinidad. Posteriormente, se cultivaron en matraces de $250 \mathrm{ml}$ por triplicado y aclimatados por 4 días. Para cada tratamiento se hicieron tres réplicas y a cada una de ellas se adicionaron inóculos $(5 \mathrm{ml})$ de $0.27 \times 10^{6} \mathrm{cel} / \mathrm{ml}$ (T1), $0.33 \times 10^{6} \mathrm{cel} / \mathrm{ml}$ (T2), $0.37 \times 10^{6} \mathrm{cel} / \mathrm{ml}$ (T3), $0.33 \times 10^{6} \mathrm{cel} / \mathrm{ml}$ (T4) y $0.40 \times 10^{6} \mathrm{cel} / \mathrm{ml}$ (T5). Seguidamente se cultivaron en matraces de 500 y $1000 \mathrm{ml}$ por siete días. Cada matraz fue sometido a aireación continua mediante una compresora magnética de aire Resum R ACO-003 a una presión de $0.027 \mathrm{MPa}$. Los cultivos fueron mantenidos bajo condiciones de laboratorio: temperatura $\left(27 \pm 2{ }^{\circ} \mathrm{C}\right)$, intensidad de luz $(1250 \pm 101 \mathrm{x})$ y fotoperiodo $(24: 0 \mathrm{~h})$.

La temperatura fue medida con un termómetro de mercurio convencional (-10 a $120^{\circ} \mathrm{C}$ ), el pH con un potenciómetro portátil OaktunpHTestr 1, previamente calibrado con amortiguador de 7.0 y 10.0 y la iluminación con un fotómetro Fisher Scientific. Las variables monitoreadas cada 24 horas fueron temperatura, iluminación, $\mathrm{pH}$ y densidad celular. 


\section{Crecimiento Poblacional Algal}

La densidad celular (células/ml) se evaluó diariamente mediante contajes celulares en cámara de Neubauer de $0.1 \mathrm{~mm}$ de profundidad hasta alcanzar el inicio de la fase estacionaria (día 20), donde se retiró la aireación de las unidades experimentales a fin de inducir la sedimentación de la biomasa por espacio de tres días. Luego, la biomasa se separó del sobrenadante por decantación y se colocó extendida en placas petri para su secado al sol. La velocidad de crecimiento en la fase exponencial y el tiempo de duplicación fueron calculados según Wood et al. (2005), de la siguiente manera:

- Tasa de crecimiento de la población $(r)=$ Ln $\left(\mathrm{N}_{\mathrm{t}} / \mathrm{N}_{\mathrm{o}}\right) / \Delta \mathrm{t}$, donde $\mathrm{N}_{\mathrm{o}}=$ tamaño de la población al inicio de un intervalo de tiempo, $\mathrm{N}_{\mathrm{t}}=$ tamaño de la población al final de un intervalo de tiempo, y $\Delta t=$ intervalo de tiempo $\left(t_{t}-t o\right)$ en días

- Duplicaciones por día $(\mathrm{k})=\log _{2}\left(\mathrm{~N}_{\mathrm{t}} / \mathrm{N}_{\mathrm{O}}\right) / \Delta \mathrm{t}$

- Tiempo de duplicación (T2) expresado en horas / división celular $\left(\mathrm{T}_{2}\right)=0.6931 / \mathrm{r}$, donde $r=$ tasa de crecimiento de la población, $0.6931=\operatorname{Ln} 2$

\section{Productividad Microalgal}

La biomasa seca fue calculada por el método de centrifugación, según Molina et al. (2003), mientras que el total de clorofilas y las biomasas se calcularon según lo establecido por Nakanishi y Deuchi (2014). A cada fracción volumétrica se le determinó la turbidez; posteriormente, se colocó en un crisol de porcelana, se secó la muestra en un deshidratador a $60{ }^{\circ} \mathrm{C}$, se atemperó en un desecador a temperatura ambiente $\mathrm{y}$, finalmente, se pesó en una balanza analítica OHAUS de $10 \mathrm{mg}$ de precisión. La identidad de las clorofilas fue analizada por espectrofotómetro UV-2102C UNIC (longitudes de onda de máxima absorción: clorofila a 408 y $662 \mathrm{~nm})$.

\section{Análisis Estadístico}

Los datos del contaje celular (cel $/ \mathrm{ml}$ ), biomasa microalgal (mg/l) y clorofila $(\mathrm{mg} / \mathrm{l})$ se analizaron mediante análisis de varianza con $\alpha=0.05$, además de la prueba post hoc Tukey para la formación de subconjuntos homogéneos entre tratamientos. Los datos fueron procesados utilizando los programas Microsoft Excel $^{\circledR} 2010$ e IBM SPSS ${ }^{\circledR}$ v. 20.

\section{Resultados}

La cepa nativa $C$. peruviana se desarrolló en todas las salinidades evaluadas durante la fase exponencial (Cuadro 1; Figura 1). Así mismo, se observó una rápida adaptación de la microalga al cambio de salinidad en los medios de cultivos anéxicos y discontinuos. Los análisis estadísticos $(\mathrm{p}<0.05)$ demostraron que los valores promedio de la densidad, biomasa seca y clorofila difieren significativamente entre los niveles de salinidad.

\section{Densidad Celular}

La cepa presentó un crecimiento más lento ante mayores niveles de salinidad (Cuadro 1). Los valores promedios de densidad celular crecieron cada día en todos los niveles de salinidad y fueron más elevados en la cepa cultivada a salinidades de $38 \%$ $\left(42.3 \pm 2.6 \times 10^{6} \mathrm{cel} / \mathrm{ml}\right)$ y $44 \%$ o $\left(37.2 \pm 2.2 \times 10^{6}\right.$ $\mathrm{cel} / \mathrm{ml}$ ). En cambio, la densidad celular descendió en un 19.2 y $19.4 \%$ respecto a la máxima obtenida en las salinidades de 59 y $88 \%$. El menor promedio de la densidad celular $\left(16.1 \pm 1.4 \times 10^{6} \mathrm{cel} / \mathrm{ml}\right)$ correspondió a la cepa cultivada a la salinidad de $176 \%$, resultado que fue significativamente inferior $(\mathrm{p}<0.05)$ con respecto a la salinidad de $38 \%$.

\section{Velocidad de Crecimiento}

La salinidad mostró un efecto significativo $((\mathrm{F}=4.904 ; \mathrm{p}<0.05)$ sobre la velocidad de 
Cuadro 1. Valores promedios diarios de crecimiento (cel x 106/ml) de Chorella peruviana en la fase exponencial (19 días) en medio de cultivo $\mathrm{f} / 2$ a cinco niveles de salinidad (datos expresados como media \pm desviación estándar; $\mathrm{n}=3$ )

\begin{tabular}{|c|c|c|c|c|c|}
\hline \multirow{2}{*}{ Día } & \multicolumn{5}{|c|}{ Salinidad } \\
\hline & $38 \%$ & $44 \%$ & $59 \%$ & $88 \%$ & $176 \%$ \\
\hline 1 & $0.7 \pm 0.1$ & $0.7 \pm 0.15$ & $0.8 \pm 0.1$ & $0.6 \pm 0.1$ & $0.57 \pm 0.1$ \\
\hline 2 & $0.8 \pm 0.1$ & $0.9 \pm 0.2$ & $0.9 \pm 0.15$ & $0.9 \pm 0.2$ & $0.6 \pm 0.2$ \\
\hline 3 & $1.5 \pm 0.2$ & $2.1 \pm 0.17$ & $2.2 \pm 0.1$ & $1.4 \pm 0.1$ & $0.6 \pm 0.1$ \\
\hline 4 & $3.2 \pm 0.3$ & $3.6 \pm 0.2$ & $3.2 \pm 0.18$ & $1.9 \pm 0.2$ & $0.7 \pm 0.1$ \\
\hline 5 & $6.3 \pm 0.1$ & $6.2 \pm 0.1$ & $4.8 \pm 0.1$ & $2.5 \pm 1.0$ & $0.8 \pm 0.1$ \\
\hline 6 & $9.3 \pm 0.1$ & $8.1 \pm 0.1$ & $6.1 \pm 0.2$ & $3.9 \pm 0.1$ & $0.9 \pm 0.1$ \\
\hline 7 & $12.2 \pm 0.2$ & $10.2 \pm 0.1$ & $8.3 \pm 0.2$ & $5.7 \pm 0.2$ & $1.1 \pm 0.2$ \\
\hline 8 & $16.6 \pm 0.2$ & $12.5 \pm 0.1$ & $11.1 \pm 0.1$ & $8.4 \pm 0.3$ & $1.4 \pm 0.1$ \\
\hline 9 & $19.7 \pm 0.2$ & $16.1 \pm 0.2$ & $14.4 \pm 0.3$ & $10.1 \pm 1.0$ & $2.1 \pm 0.2$ \\
\hline 10 & $22.8 \pm 0.1$ & $19.5 \pm 0.1$ & $17.9 \pm 0.1$ & $12.0 \pm 0.1$ & $3.2 \pm 0.1$ \\
\hline 11 & $26.6 \pm 0.1$ & $23.3 \pm 0.2$ & $21.5 \pm 0.2$ & $13.6 \pm 0.1$ & $4.3 \pm 0.1$ \\
\hline 12 & $30.2 \pm 0.1$ & $26.7 \pm 0.1$ & $25.4 \pm 0.1$ & $16.7 \pm 1.0$ & $5.2 \pm 0.1$ \\
\hline 13 & $32.2 \pm 0.3$ & $30.1 \pm 0.1$ & $28.1 \pm 0.1$ & $20.6 \pm 0.2$ & $6.6 \pm 0.1$ \\
\hline 14 & $34.9 \pm 0.2$ & $33.5 \pm 0.1$ & $31.7 \pm 0.1$ & $25.6 \pm 1.0$ & $7.9 \pm 0.1$ \\
\hline 15 & $37.9 \pm 0.1$ & $36.3 \pm 0.3$ & $33.6 \pm 0.2$ & $30.3 \pm 0.1$ & $9.0 \pm 0.1$ \\
\hline 16 & $39.7 \pm 0.2$ & $37.6 \pm 0.1$ & $34.9 \pm 0.4$ & $31.6 \pm 0.1$ & $10.9 \pm 0.1$ \\
\hline 17 & $41.2 \pm 0.1$ & $38.2 \pm 0.1$ & $35.2 \pm 0.1$ & $32.4 \pm 0.2$ & $12.8 \pm 0.1$ \\
\hline 18 & $41.9 \pm 0.8$ & $38.2 \pm 0.1$ & $35.5 \pm 0.1$ & $35.3 \pm 0.1$ & $14.5 \pm 0.1$ \\
\hline 19 & $42.2 \pm 2.6$ & 37. $2 \pm 2.2$ & 34. $2 \pm 2.1$ & $34.2 \pm 1.8$ & $16.1 \pm 1.4$ \\
\hline
\end{tabular}

crecimiento y tiempo de duplicación en la fase exponencial de la cepa nativa de $C$. peruviana (Cuadro 2). En la salinidad de $38 \%$ se observó el mayor crecimiento con valores de $\mathrm{r}=0.61, \mathrm{~K}=0.88$ y $\mathrm{T} 2=1.14 \mathrm{~h}$ por división celular en los cultivos. Por otro lado, en otros regímenes de salinidad, el crecimiento algal se mantuvo próximo a la velocidad de crecimiento y tiempo de duplicación, con excepción de la salinidad de $17 \%$, donde la población celular creció moderadamente con valores $\mathrm{r}=0.20, \mathrm{~K}=0.28$ y T2 $=3.51 \mathrm{~h}$ por división celular.
El análisis de la velocidad de crecimiento mostró que las tasas de crecimiento de población (r) y la duplicación por día (k) se van reduciendo conforme aumenta la salinidad, de modo que se puede afirmar que existe una relación significativa $(\mathrm{p}<0.05)$ entre el tiempo de duplicación en horas/división celular (t2) y salinidad.

\section{Productividad: Biomasa y Clorofila}

El máximo valor de productividad de biomasa seca se obtuvo con la salinidad de $38 \%$ con un valor de $397.9 \pm 2.3 \mathrm{mg} / 1$ 
Cuadro 2. Velocidad de crecimiento y tiempo de duplicación obtenidos en la fase exponencial de los cultivos de C. peruviana a cinco niveles de salinidad

\begin{tabular}{lccccc}
\hline & $38 \% 0$ & $44 \% 0$ & $59 \% 0$ & $88 \% 0$ & $176 \% 0$ \\
\hline $\begin{array}{l}\text { Tasa de crecimiento de la } \\
\text { población (r) }\end{array}$ & 0.61 & 0.55 & 0.54 & 0.42 & 0.20 \\
$\begin{array}{l}\text { Duplicación por día (k) } \\
\begin{array}{l}\text { Tiempo de duplicación (t2) } \\
\text { (división celular/h) }\end{array}\end{array}$ & 0.88 & 0.79 & 0.79 & 0.60 & 0.28 \\
\hline
\end{tabular}

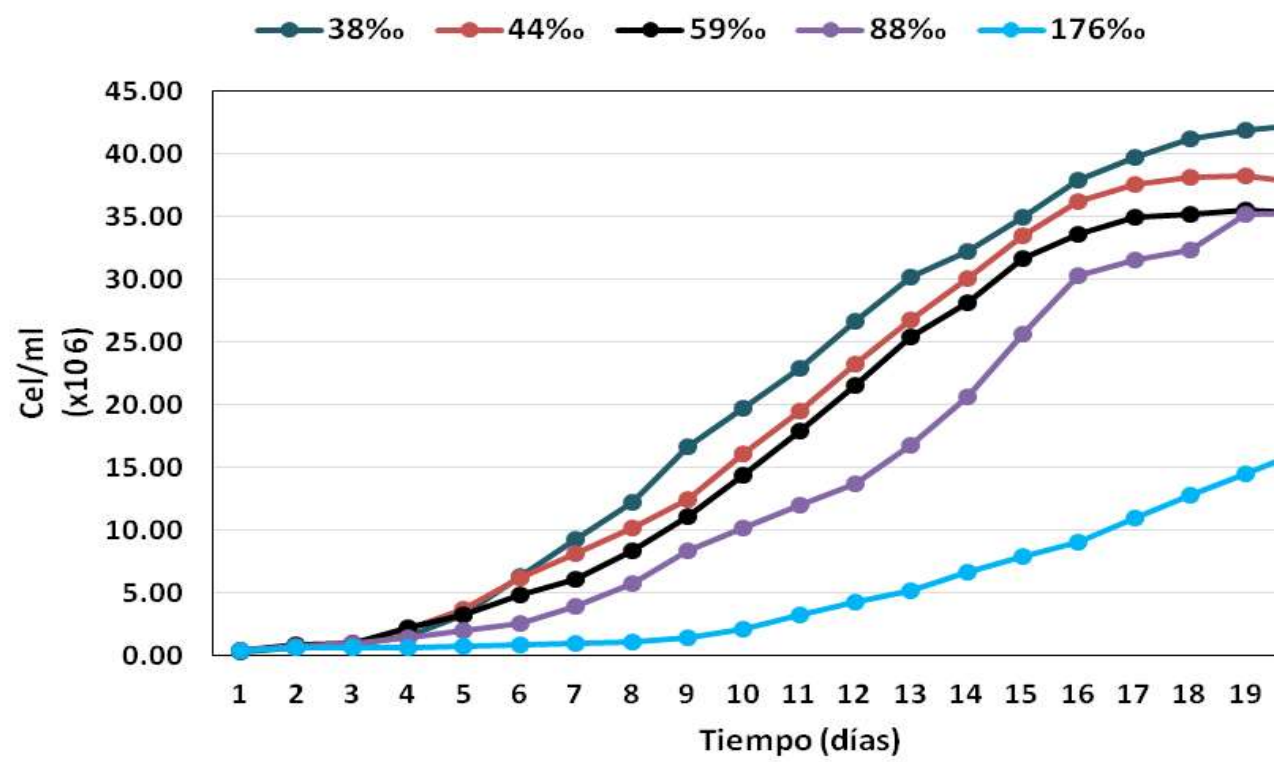

Figura 1. Crecimiento (cel $\times 10^{6} / \mathrm{ml}$ ) de Chorella peruviana en medio de cultivo f/2 a cinco niveles de salinidad evaluados durante la fase exponencial (19 días)

$(\mathrm{p}<0.05)$. Las salinidades de 44 y $59 \%$ presentaron valores próximos a la salinidad de $38 \%$ o $(390.5 \pm 2.3$ y $389.6 \pm 2.3 \mathrm{mg} / 1$, respectivamente) (Figura 2).

Asimismo, hubo diferencia significativa $(\mathrm{p}<0.05)$ entre los valores promedio de la clorofila durante la fase exponencial. Las salinidades de 38 y 44\%o presentaron los mayores contenidos de clorofila total: $80.2 \pm$ $2.3 \mathrm{mg} / 1$ y $76.7 \pm 1.5 \mathrm{mg} / 1$, respectivamente; mientras que con $59 \%$ de salinidad se encon- tró un valor de $75.7 \pm 2.4 \mathrm{mg} / \mathrm{l}$, y con la salinidad de $176 \%$ solo se llegó a obtener 65.8 $\pm 1.3 \mathrm{mg} / \mathrm{l}$ de biomasa seca de clorofila.

\section{Discusión}

Los resultados confirman que la cepa nativa de $C$. peruviana de la laguna salina superficial de Chilca es eurihalina, lo que indica que la microalga se adapta rápidamente 


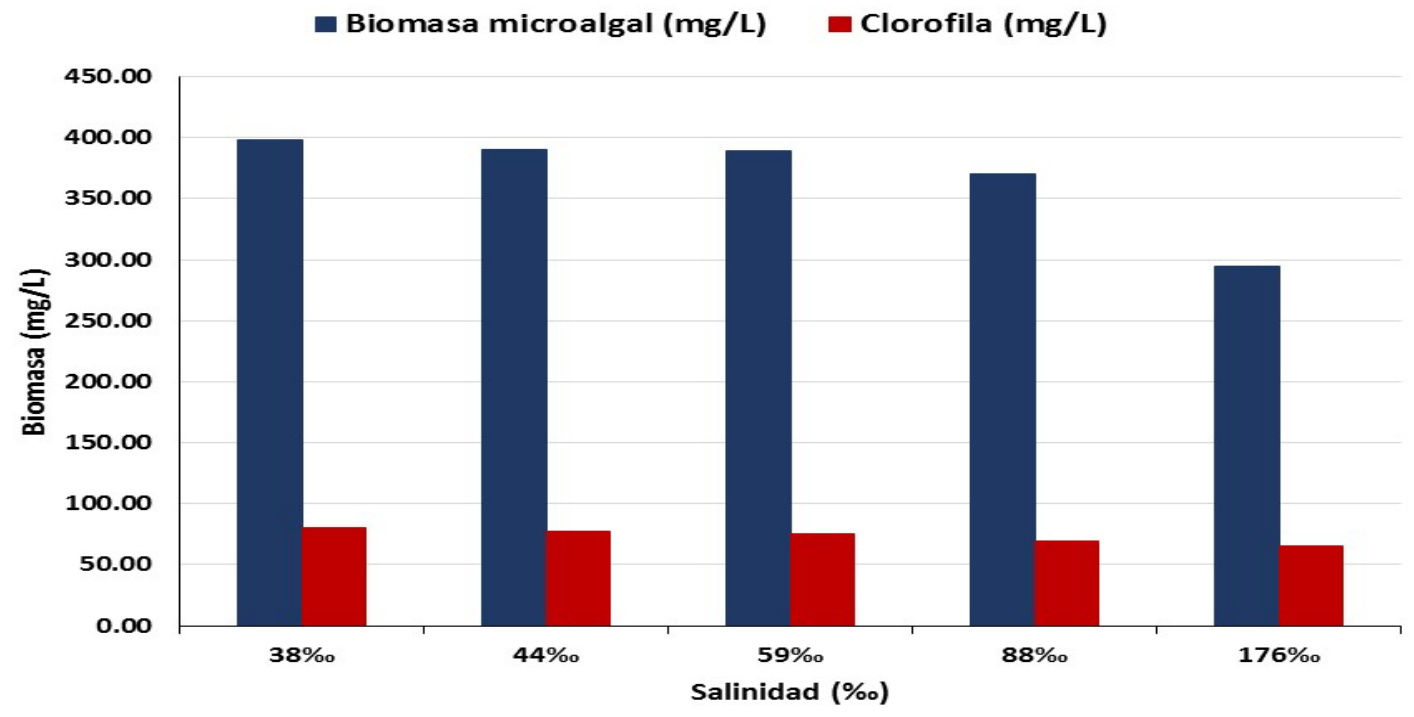

Figura 2. Productividad y clorofila ( $\mathrm{mg} / \mathrm{l})$ de cultivos de Chlorella peruviana en cinco niveles de salinidad

al cambio de salinidad; en este caso, desde una salinidad de $38 \%$ hasta $176 \%$ desde el primer día hasta los 19 días de la siembra del inóculo (Cuadro 1; Figura 1). Esta condición también puede ser atribuida al estado de desarrollo, al sistema de cultivo, cantidad y tipo de alimento y, consecuentemente, a las condiciones de manejo.

De acuerdo a las fuentes de nutrientes del medio de cultivo, se ha demostrado que el medio $\mathrm{f} / 2$ (Guillard) mejora el crecimiento y mantiene viable la cepa nativa. Diversos reportes indican que este medio nutritivo en cultivo discontinuo contribuye a una mayor productividad y crecimiento en distintas especies de microalgas, cuando se trata con cultivos en medios no salinos y salinos (Markou et al., 2014; Ramaraj et al., 2015). Chacón (1980) formuló dos medios de cultivo para crecimiento microalgal teniendo como base el agua de las salinas de Chilca y un medio artificial similar a la composición del agua de la laguna. Calvo-Perez y Molinari (2015) indicaron que el medio de cultivo Calderón (Serpa y Calderón, 2005) con $\mathrm{NaCl}$ saturado puede ser utilizado para especies microalgales de ambientes hipersalinos, vali- dándolo en $C$. peruviana al encontrar una alta densidad en sus cultivos, aunque no llegaron a reportar sus valores.

La salinidad del medio de cultivo ejerció una disminución en las densidades celulares. En la laguna salinas de Chilca, situada a $100 \mathrm{msnm}$, la salinidad es variable, registrándose entre $30.25 \%$ y $117.22 \%$ (Chacón, 1980), debido al origen del sistema acuático que se encuentra influenciado por aguas saladas subterráneas. El 38\%o es el promedio de salinidad del sistema, valor que coincidió con la máxima producción de las tasas de crecimiento poblacional de la cepa nativa. Este aspecto es muy importante para una posible aplicación práctica y trabajos específicos en lagunas con salinidades superiores a los del mar, pues allí existe una baja diversidad de organismos por la adaptación a la osmoregulación.

Cabe señalar que diversos autores reportan que ciertas cepas de Chlorella tienen crecimientos óptimos entre 40 y $180 \%$ de salinidad (Kessler y Huss, 1992; Huss et al., 1999; Wehr y Sheath, 2003; Xu et al., 2006; Chinnasamy et al., 2010). De igual forma, 
Moronta et al. (2006) demostraron que las cepas de $C$. sorokiniana tuvieron una tendencia decreciente en relación a las salinidades. Es importante considerar que Chlorella es un género de microalgas clorofíceas que se caracteriza por presentar un rápido crecimiento en cultivo celular y por su capacidad de utilizar como sustrato nutricional compuestos orgánicos e inorgánicos (Wehr y Sheath, 2003; Xu et al., 2006; Chinnasamy et al., 2010; Sing y Sharma, 2014).

A diferencia de lo reportado en este trabajo, Emad et al. (2015) reportan que $C$. vulgaris tuvo crecimientos máximos de $30 \times 10^{6}$ a $125 \times 10^{6} \mathrm{cel} / \mathrm{ml}$ para la salinidad de $25 \%$ enriquecida con el medio de cultivo $\mathrm{f} / 2$. Mora et al. (2005) señala para Chlorella sp, aislada de las aguas de la represa de Tulé (Venezuela) una densidad de $79.51 \times 10^{6} \mathrm{cel} / \mathrm{ml}$ en los cultivos controles con agua potable no destilada y con medio $\mathrm{f} / 2$; en cambio cuando fue cultivada a 25 y $35 \%$ de salinidad disminuyó la densidad celular de $1.75 \times 10^{6}$ a $1.47 \times 10^{6} \mathrm{cel} / \mathrm{ml}$. Moronta et al. (2006) demostraron que las cepas de $C$. sorokiniana presentan densidades celulares en medio no salino con $59.18 \times 10^{6} \mathrm{cel} / \mathrm{ml}$ para los cultivos no axénicos y de 109.11 y $89.70 \times 10^{6} \mathrm{cel} / \mathrm{ml}$ en cultivos mixotróficos y heterotróficos, respectivamente. Otros autores, han demostrado que otras cepas de Chlorella aisladas de ambientes marinos y salobres presentan bajas densidades celulares. Así, Illman et al. (2000) encontraron una densidad promedio de $6 \times 10^{6} \mathrm{cel} / \mathrm{ml}$ y una biomasa de $460 \mathrm{mg} / \mathrm{l} \mathrm{a}$ partir del uso del medio f/2; Shah et al. (2003) lograron obtener un crecimiento de $16 \times 10^{6}$ cel $/ \mathrm{ml}$ utilizando el sistema $\mathrm{f} / 2$; y Cao et al. (2014) mencionan que la mayor biomasa en cultivos con el medio $\mathrm{f} / 2$ lo obtienen a una salinidad del $20 \%$.

En cada especie de Chlorella, la concentración de biomasa debe variar en relación al rango de tolerancias de salinidades y al medio de cultivo (Wehr y Sheath, 2003; Xu et al., 2006; Chinnasamy et al., 2010). Por estos motivos, las especies del género Chlorella han sido ampliamente estudiadas para incluirlas en procesos biotecnológicos que generen productos destinados a las industrias alimenticias, farmacéuticas, de saneamiento ambiental, agropecuarias y energéticas (Chisti, 2007). Específicamente $C$. sorokiniana posee un gran potencial para la producción de ácidos grasos omega 3, 6 y 9; además de la producción de biocombustibles (Spolaore et al., 2006; Chisti, 2007; Sing y Goyal, 2007). Los valores extremos de salinidades afectan negativamente las tasas de fotosíntesis, respiración y crecimiento de las microalgas marinas (Bermúdez et al., 2002; Helm et al., 2006).

La salinidad del medio de cultivo ejerció una relación negativa en las tasas de crecimiento y tiempo de duplicación en la fase exponencial de la cepa nativa de $C$. peruviana, debido al hecho que las células disminuyen la duplicación con el aumento de la salinidad, comportamiento observado en otros estudios (Ortiz et al., 2012; Emad et al., 2015).

La mayor biomasa seca y contenido de clorofila en los cultivos fue obtenida en los cultivos de 38\%o de salinidad (Cuadro 3). El efecto de elevadas salinidades sobre la biomasa seca y clorofila se ha reportado en Chlorella sp, cepa nativa aislada de la represa de Tule, Venezuela, con un incremento en biomasa y clorofila de hasta $70 \%$ (Mora et al., 2005). Este resultado obedece al hecho que las células disminuyen la velocidad de crecimiento con la salinidad, con lo cual permanecen de mayor tamaño respecto a las células de menor tamaño no sometida a salinidades, las cuales exhiben la menor tasa de duplicación (Latala, 1991; Hiremath y Mathad, 2010).

C. peruviana tiene una alta tolerancia por las aguas salinas y aparenta ser una buena candidata para sistemas de cultivo en agua de mar y salobre. Su cultivo parece factible en salinidades hasta al menos $176 \%$. 
Chlorella es considerada como una microalga eurihalina con una mayor tolerancia a las altas concentraciones de sal en el agua que la Chlorella vulgaris (Nakanishi y Deuchi, 2014).

\section{Conclusiones}

- La microalga nativa Chlorella peruviana demostró factibilidad de cultivo y capacidad de crecimiento entre 38 y $176 \%$ de salinidad, con una óptima a $38 \%$, mientras que el contenido celular de biomasa seca y de clorofila presenta un máximo en el nivel de $38 \%$ de salinidad. Sin embargo, es necesario probar salinidades en menores concentraciones para fines comparativos.

- La salinidad influye negativamente sobre el crecimiento, biomasa y contenido de clorofila de la microalga.

\section{Agradecimientos}

El presente estudio fue parcialmente financiado por el proyecto N..$^{\circ} 132$-FINCyTIB-2013. Los autores manifiestas su agradecimiento a la Universidad Nacional Mayor de San Marcos por el apoyo en la coordinación de esta investigación.

\section{Literatura Citada}

1. Adenan NS, Yusoff FM, Shariff M. 2013. Effect of salinity and temperature on the growth of diatoms and green algae. J Fish Aquat Sci 8: 397-404. doi: 10.3923/jfas.2013.397.404

2. Alvarez CM, Gallardo T. 1989. Una revisión sobre biotecnología de las algas. Bot Complitensis 15: 9-60.

3. Alveal K. 1995. Manejo de algas marinas. En: Alveal K, Ferrario ME, Oliveira EC, Sar E (eds). Manual de métodos ficológicos. Concepción, Chile: Universidad de Concepción. p 825-863.
4. Bermúdez, JC, Lodeiros J, Morales E. 2002. Producción de biomasa de la microalga marina Chroomonas sp, en función del $\mathrm{pH}$, intensidad luminosa y salinidad. Bol Inv Mar Cost 31: 167-185.

5. Calvo-Pérez J, Molinari E. 2015. A nomenclatural and cultural note on Chlorella peruviana G. Chacón and other species of the genus Chlorella Beij (Chlorellaceae, Chlorellales). Biologist (Lima) 13(1): 153-156.

6. Cao J, Yuan HL, Li BZ, Yang JS. 2014. Significance evaluation of the effects of environmental factors on the lipid accumulation of Chlorella minutissima UTEX 2341 under lownutrition heterotrophic condition. Bioresource Technol 152: 177-184. doi: 10.1016/j.biortech.2013.10.084

7. Chacón RG. 1980. Chlorella peruviana sp. nov. y su ambiente altamente salino. Bol Soc Per Botánica 8: 83-96.

8. Chinnasamy S, Bhatnagar A, Hunt RW, Das KC. 2010. Microalgae cultivation in a wastewater dominated by carpet mill effluents for biofuel applications. Bioresource Technol 101: 3097-3105. doi: 10.1016/j.biortech.2009.-12.026

9. Chisti Y. 2007. Biodiesel from microalgae. Biotechnol Adv 25:294-306. doi: 10.1016/j.biotechadv.2007.02.001

10. Emad AM, Salarzadeh A, Fourooghifard H. 2015. Comparison of salinity effects and medium of TMRL, Guillard and Comway cultivation on density and bloom of Chlorella vulgaris alga in vitro. Int J Adv Res 3: 1236-1243.

11. Graeff A. 2003. Método para multiplicacao da alga (Chlorella minutissima) para alimentacao inicial de um sistema de producao de peixes fitoplantofagos. In: Congreso Iberoamericano Virtual de Acuicultura. Zaragoza, España.

12. Guillard RRL. 1975. Culture of phytoplankton for feeding marine invertebrates. In: Smith WL, Chanley MH (eds). Culture of marine inverte- 
brate animals. New York: Plenum Press. p 26-60.

13. Helm MMN, Bourne A, Lovatelli A. 2006. Cultivo de bivalvos en criadero. Un manual práctico. FAO Documento Técnico de Pesca 471. Roma: FAO. 184 p.

14. Hernandez PA, Labbé JI. 2014. Microalgas, cultivo y beneficios. Rev Biol Mar Oceanogr 49: 157-173. doi: 10.4067/ S0718-19572014000200001

15. Hiremath S, Mathad P. 2010. Impact of salinity on the physiological and biochemical traits of Chlorella vulgaris Beijerinck. J Algal Biomass Utln 1: 51-59.

16. Huss VA, Frank C, Hartmann E, Hirmer M, Kloboucek A, Seidel B, et al. 1999. Biochemical taxonomy and molecular phylogeny of the genus Chlorella sensu lato (Chlorophyta). J Phycol 35: 587-598. doi: 10.1046/j.15298817.1999.3530587.x

17. Illman AM, Scragg AH, Shales SW. 2000. Increase in Chlorella strains calorific values when grown in low nitrogen medium. Enzyme Microb Tech 27: 631-635. doi: 10.1016/S01410229(00)00266-0

18. Infante CH, Angula E, Zarate A, Florez I, Barrios F, Zapata C. 2012. Propagación de la microalga Chlorella sp en cultivo por lote: cinética de crecimiento celular. Av Cienc Ing 3: 159-164.

19. Kessler E, Huss, VA. 1992. Comparative physiology and biochemistry and taxonomic assignment of the Chlorella (Chlorophyceae) strains of the Culture Collection of the University of Texas at Austin. J Phycol 28: 550-553. doi: 10.1111/j.0022-3646.1992.00550.x

20. Kirst GO. 1989. Salinity tolerance of eukaryotic marine algae. Annu Rev Plant Phys 40: 21-53.

21. Krienitz L, Huss V, Bock C. 2015. Chlorella: 125 years of the green survivalist. Trends Plant Sci 20: 67-69. doi: $10.1016 /$ j.tplants.2014.11.005

22. Latala A. 1991. Effects of salinity, temperature and light on the growth and morphology of green planktonie algae. Oceanologia 31: 119-138.
23. Markou G, Vandamme D, Muylaert K. 2014. Microalgal and cyanobacterial cultivation: the supply of nutrients. Water Res 65: 186-202. doi: 10.1016/ j.watres.2014.07.025

24. Mathad P, Hiremath SH. 2009. Alleviation of saline stress by gypsum in Chlorella vulgaris Beijerinck. J Algal Biomass Utln 1: 43-53.

25. Molina Grima E, Belarbi E, Acién Fernandez, F, Robles Medina A, Chisti Y. 2003. Recovery of microalgal biomass and metabolites: process options and economics. Biotechnol Adv 20: 491515. doi: 10.1016/S0734-9750(02)00050-2

26. Montoya H, Espinoza J. 1985. Algas de las lagunas de oxidación de Ventani1la, Perú. Bol Lima 47(7): 41-68.

27. Mora R, Moronta R, Ortega J, Morales $\boldsymbol{E}$. 2005. Crecimiento y producción de pigmentos de la microalga nativa Chlorella sp aislada de la Represa de Tulé, Municipio Mara, Estado Zulia, Venezuela. Ciencia (Maracaibo) 12: 117-124.

28. Moronta R, Mora R, Morales E. 2006. Respuesta de la microalga Chlorella sorokiniana al $\mathrm{pH}$, salinidad y temperatura en condiciones axénicas y no axénicas. Rev Fac Agron 23: 27-41.

29. Nakanishi K, Deuchi K. 2014. Culture of a high-chlorophyll-producing and halotolerant Chlorella vulgaris. J Biosci Bioeng 117: 617-619. doi: 10.1016/ j.jbiosc.2013.10.024

30. Ortiz MM, Cortés CC, Sánchez VJ, Padilla J, Otero PA. 2012. Evaluación del crecimiento de la microalga Chlorella sorokiniana en diferentes medios de cultivo en condiciones autotróficas y mixotróficas. Orinoquia 16: 11-20.

31. Ramaraj R, Tsai DD, Chen PH. 2015. Biomass of algae growth on natural water medium. J Photoch Photobio B 142: 124-128. doi: 10.1016/j.jphotobiol.2014.12.007

32. Rodas GH, Rodríguez FH, Flores MG, Vidales CJ, Aranda RJ, Luna MA. 2012. Efecto de la densidad celular de inoculación en el crecimiento de 
Chlorella vulgaris CLV2 cultivada bajo condiciones mixotroficas. Rev Fitotec Mex 35 (Núm Esp 5): 83-86.

33. Rodrigues JB, Belli-Filho PE. 2004. Eficiência da microalga Chlorella minutissima no tratamento de residuos de suinocultura enriquecido com uréia. Biotemas 17: 7-26.

34. Shah MM, Alam MJ, Mia MY. 2003. Chlorella sp: isolation, pure culture and small scale culture in brackish-water. Bangladesh J Sci Ind Res 38: 165-174.

35. Serpa IRF, Calderón RA. 2005. Efecto del estrés por salinidad en cuatro cepas de Dunaliella salina Teod en el Perú. Ecol Aplicada 4: 127-133.

36. Singh S, Goyal D. 2007. Microbial and plant derived biomass for removal of heavy metals from wastewater. Bioresoursce Technol 98: 2343-2357. doi: 10.1016/j.biortech.2005.12.006

37. Singh UB, Sharma C. 2014. Determination of suitable growth medium for Chlorella minutissima. Int $\mathrm{J}$ Engineer Sci Res Technol 3: 481-485.

38. Spolaore P, Joannis-Cassan C, Duran E, Isambert A. 2006. Commercial applications of microalgae. J Biosci Bioeng 101: 87-96. doi: 10.1263/jbb.101.87
39. Vincens A, Chacón G, Popovici Z. 1971. Contribución al conocimiento de las algas de las lagunas de bioestabilización en San Juan. Bol Ofic Sanit Panam 70: 148-164.

40. Wehr J, Sheath R. 2003. Freshwater algae of North America: ecology and classification. San Diego, USA: Academic Press. 1050 p.

41. Wood A, Everroad R, Wingard $L$. 2005. Measuring growth rates in microalgal cultures. In: Andersen R (ed). Algal culturing techniques. USA: Elsevier, Academic Press. p 269-286.

42. Хи H, Miao $X, W u$ Q. 2006. High quality biodiesel production from a microalga Chlorella protothecoides by heterotrophic growth in fermenters. $\mathrm{J}$ Biotechnol 126: 499-507. doi: 10.1016/ j.jbiotec.2006.05.002

43. Yamamoto M, Fujishita M, Hirata A, Kawano S. 2004. Regeneration and maturation of daughter cell walls in the autospore-forming green alga Chlorella vulgaris (Chlorophyta, Trebouxiophyceae). J Plant Res 117: 257-264. doi: 10.1007/s10265-004-0154-6 